The copper-silver system was chosen as a crucial test of the method because the existence of a eutectic is not in agreement with the Hume-Rothery rules, which would predict complete miscibility of the two metals. Four alloys containing 23.0, 39.9 (eutectic composition), 62.9 , and 88.5 at. $\%$ copper were quenched from melt. The $\mathrm{x}$-ray diffraction patterns of these alloys showed a single phase. On a plot of unit cell size vs concentration, the lattice parameters obtained with the four metastable alloys fell on a smooth curve connecting the two previously known segments of curves within the solubility limits at both ends of the phase diagram (14.1 and 95.1 at. $\% \mathrm{Cu}$ ). The maximum deviation from linearity (Vegard's law) was less than $1 \%$ at $50 \% \mathrm{Cu}$. Such a deviation is not unusual in solid solution binary alloys. These results establish without doubt that, during solidification, the two stable phases, copper rich and silver rich, did not have time to nucleate and grow, and metastable solid solutions were obtained.

* This work was jointly sponsored by the Atomic Energy Commission and the Office of Naval Research.

1 W. T. Olsen, Jr, and Ralph Hultgren, Trans. AIME, 188, 1223 (1950)

\section{Metastable Electron Compound in Ag-Ge Alloys}

Pol Duwez, R. H. Willens, AND W. Klement, JR. Division of Engineering, California Institute of Technology, Pasadena, Californio

(Received February 19, 1960)

$I^{\mathrm{N}}$ the previous Letter to the Editor, ${ }^{1}$ the existence of a continuous series of metastable solid solutions in $\mathrm{Cu}-\mathrm{Ag}$ alloys was reported. By quenching the molten alloys rapidly enough, the normal processes of nucleation and growth were prevented and solid solutions were obtained. These metastable solid solutions were predictable since copper and silver satisfy the criteria for complete solid solubility proposed by Hume-Rothery.

In Ag-Ge alloys complete solid solubility is precluded because of dissimilar crystal structures. The Ag-Ge equilibrium phase diagram $^{2}$ is of the eutectic type with a maximum solubility of germanium in silver of 9.6 at.\% and negligible solubility of silver in germanium. The eutectic composition is at 25.9 at.\% Ge. It was expected that the solubility limits on both ends of the phase diagram would be extended by rapid quenching. An alloy containing 25.7 at. $\%$ Ge was quenched from the melt. This alloy had a crystal structure that was neither face-centered cubic nor diamond cubic, but rather hexagonal close-packed with $a=2.987 \pm 0.003, c=4.716 \pm 0.002$, and $c / a=1.628 \pm 0.003$. The presence of a small amount of a diamond cubic germanium phase was indicated by a few weak diffraction peaks on the $x$-ray pattern. The new metastable hexagonal phase is considered to be an electron compound with an electron concentration of $7 / 4$ electrons/atom.

In 1940, Hume-Rothery et al..$^{3}$ suspected the existence of a $\frac{3}{2}$ electron compound in Ag-Ge alloys, but failed to detect it under equilibrium conditions. In order to determine if such a compound could be synthesized by rapid quenching, an alloy containing 16.4 at. $\%$ Ge was investigated. No body-centered cubic phase was found, but again a hexagonal close-packed structure occurred with $a=2.891 \pm 0.002, c=4.718 \pm 0.006$, and $c / a=1.632 \pm 0.003$. The existence of the hexagonal structure instead of the bodycentered cubic structure at the electron concentration of $\frac{3}{2}$ has been noted in other alloy systems. Satisfactory explanations for the occurrence of a hexagonal close-packed structure in the region of a $\frac{3}{2}$ electron concentration have not been advanced. It might be noted, however, that when a $\frac{3}{2}$ electron compound ( $\beta$-phase) does exist, it is usually more stable at high temperatures. It is probable that the electronic configurational energy is not the main factor in creating a $\frac{3}{2}$ electron compound ( $\beta$-phase). It is suggested that the high-lattice entropy contribution, char- acteristic of a body-centered cubic structure, has a prominent role in lowering the free energy of this phase at high temperatures.

The interpretation of the structure of alloys containing less than 16.4 at.\% Ge has not been completed. It appears, however, that the solubility of germanium in face-centered cubic silver is extended beyond 9.6 at. $\%$ Ge. In the alloys containing more than 25.7 at. \% Ge, both the hexagonal compound and diamond cubic germanium were found. No measurable change in the lattice parameter of the diamond cubic germanium could be observed. Because of the nearly equal size of silver and germanium (as deduced from the change in lattice parameter at the silverrich end of the phase diagram), ${ }^{4}$ and the openness of the diamond cubic lattice, silver could probably be retained in the germanium structure without any appreciable change in the cell size. Therefore, measurements other than the lattice parameter will be necessary to establish whether there is any significant solubility of silver in germanium.

1 P. Duwez, R. H. Willens, and W. Klement, Jr., J. Appl. Phys. 31, 1136 (1960)

M. Hansen, Constitution of Binary Alloys (McGraw-Hill Book Company, Inc., New York, 1958).

3 W. Hume-Rothery, G. V. Raynor, P. W. Reynolds, and H. K. Packer J. Inst. Metals 66, 209 (1940)

4 E. A. Owen and V. W. Rowlands, J. Inst. Metals 66, 361 (1940).

\section{Erratum : Motion of High-Speed Arc Spots in Magnetic Fields}

D. ZEI AND J. G. WINANS

University of Wisconsip, Madison, Wisconsin

[J. Appl. Phys. 30, 1813 (1959).]

$I^{1}$

$N$ Fig. 5, the $A$ at the left of the figure should be replaced by (b) and the B at the left side of the figure should be replaced by (a).

\section{Books Reviewed}

Prompt, noncritical reviews appear in this column. Critical revieres of many of the books described here will appear in Physics Today, The Review of Scientific Instruments, or American Journal of Physics.

Exploding Wires. W. G. Chace and H. K. Moore, Editors. Plenum Press, Inc., New York, 1959. Price $\$ 9.50$.

The collection of papers which were presented at the conference on the exploding wire phenomenon (April, 1959) comprises the first book published on this topic. These have been arranged into three broad groups: theoretical and experimental, shock wave, and applications and techniques. For those who are not acquainted with this field, the editor, W. G. Chace, has written an introduction which is compact and informative. In the papers which follow, many aspects dealing with the electrical, optical, spectroscopic, and radiometric observations are covered. The variety of uses of exploding wires listed and the collection of specialized techniques which have been developed, as described in this volume, will make a useful addition to the store of "knowhow" for operations in this range of very rapid discharges from high-capacity condensers, of the production of intense light sources, and of very high temperatures. 